\title{
High-Resolution Diffusion Tensor Imaging and Tractography of the Human Optic Chiasm at 9.4 T
}

Citation for published version (APA):

Roebroeck, A. F., Galuske, R., Formisano, E., Chiry, O., Bratzke, H., Ronen, I., Kim, D. S., \& Goebel, R. W. (2008). High-Resolution Diffusion Tensor Imaging and Tractography of the Human Optic Chiasm at 9.4 T. Neuroimage, 39(1), 157-168. https://doi.org/10.1016/j.neuroimage.2007.08.015

Document status and date:

Published: 01/01/2008

DOI:

10.1016/j.neuroimage.2007.08.015

Document Version:

Publisher's PDF, also known as Version of record

Document license:

Taverne

Please check the document version of this publication:

- A submitted manuscript is the version of the article upon submission and before peer-review. There can be important differences between the submitted version and the official published version of record.

People interested in the research are advised to contact the author for the final version of the publication, or visit the DOI to the publisher's website.

- The final author version and the galley proof are versions of the publication after peer review.

- The final published version features the final layout of the paper including the volume, issue and page numbers.

Link to publication

\footnotetext{
General rights rights.

- You may freely distribute the URL identifying the publication in the public portal. please follow below link for the End User Agreement:

www.umlib.nl/taverne-license

Take down policy

If you believe that this document breaches copyright please contact us at:

repository@maastrichtuniversity.nl

providing details and we will investigate your claim.
}

Copyright and moral rights for the publications made accessible in the public portal are retained by the authors and/or other copyright owners and it is a condition of accessing publications that users recognise and abide by the legal requirements associated with these

- Users may download and print one copy of any publication from the public portal for the purpose of private study or research.

- You may not further distribute the material or use it for any profit-making activity or commercial gain

If the publication is distributed under the terms of Article $25 \mathrm{fa}$ of the Dutch Copyright Act, indicated by the "Taverne" license above, 


\title{
High-resolution diffusion tensor imaging and tractography of the human optic chiasm at $9.4 \mathrm{~T}$
}

\author{
Alard Roebroeck ${ }^{\mathrm{a}, *}$ Ralf Galuske, ${ }^{\mathrm{b}, \mathrm{c}}$ Elia Formisano, ${ }^{\mathrm{a}}$ Oriana Chiry, ${ }^{\mathrm{b}, \mathrm{c}}$ Hansjürgen Bratzke, ${ }^{\mathrm{d}}$ \\ Itamar Ronen, ${ }^{\mathrm{e}, \mathrm{f}}$ Dae-shik Kim, ${ }^{\mathrm{e}, \mathrm{f}}$ and Rainer Goebel ${ }^{\mathrm{a}}$
}

\author{
${ }^{a}$ Department of Cognitive Neuroscience, Faculty of Psychology, Maastricht University, The Netherlands \\ ${ }^{\mathrm{b}}$ Max-Planck-Institute for Brain Research, Frankfurt, Germany \\ ${ }^{\mathrm{c}}$ Department of Biology, Technical University of Darmstadt, Germany \\ dinstitute of Forensic Medicine, University of Frankfurt Medical School, Germany \\ ${ }^{\mathrm{e}}$ Center for Biomedical Imaging, Department of Anatomy and Neurobiology, Boston University School of Medicine, USA \\ ${ }^{\mathrm{f}}$ Center for Magnetic Resonance Research, University of Minnesota, Minneapolis, USA
}

Received 26 March 2007; revised 10 August 2007; accepted 15 August 2007

Available online 24 August 2007

\begin{abstract}
The optic chiasm with its complex fiber micro-structure is a challenge for diffusion tensor models and tractography methods. Likewise, it is an ideal candidate for evaluation of diffusion tensor imaging tractography approaches in resolving inter-regional connectivity because the macroscopic connectivity of the optic chiasm is well known. Here, highresolution (156 $\mu \mathrm{m}$ in-plane) diffusion tensor imaging of the human optic chiasm was performed ex vivo at ultra-high field $(9.4 \mathrm{~T})$. Estimated diffusion tensors at this high resolution were able to capture complex fiber configurations such as sharp curves, and convergence and divergence of tracts, but were unable to resolve directions at sites of crossing fibers. Despite the complex microstructure of the fiber paths through the optic chiasm, all known connections could be tracked by a line propagation algorithm. However, fibers crossing from the optic nerve to the contralateral tract were heavily underrepresented, whereas ipsilateral nerve-to-tract connections, as well as tract-to-tract connections, were overrepresented, and erroneous nerve-to-nerve connections were tracked. The effects of spatial resolution and the varying degrees of partial volume averaging of complex fiber architecture on the performance of these methods could be investigated. Errors made by the tractography algorithm at high resolution were shown to increase at lower resolutions closer to those used in vivo. This study shows that increases in resolution, made possible by higher field strengths, improve the accuracy of DTI-based tractography. More generally, post-mortem investigation of fixed tissue samples with diffusion imaging at high field strengths is important in the evaluation of MR-based diffusion models and tractography algorithms.
\end{abstract}

(c) 2007 Elsevier Inc. All rights reserved.

\footnotetext{
* Corresponding author. Department of Cognitive Neuroscience, Faculty of Psychology, Maastricht University, Postbus 616, 6200 MD Maastricht, The Netherlands. Fax: +31433884125 .

E-mail address: a.roebroeck@psychology.unimaas.nl (A. Roebroeck).

Available online on ScienceDirect (www.sciencedirect.com).
}

\section{Introduction}

Diffusion tensor imaging (DTI) is a magnetic resonance imaging technique that can quantify the directional dependence of water diffusion in biological tissue. In brain white matter tissue, water diffusion is greater along the direction of axon bundles than perpendicular to it (Beaulieu, 2002). By quantifying the degree and direction of this diffusion anisotropy, DTI can be used to probe the micro-structure of white matter fiber bundles non-invasively in vivo (Basser et al., 1994). Fiber tracking or tractography refers to the derivation of area-to-area axonal connectivity from the properties of local diffusion profiles. In its simplest form, tractography amounts to following the local primary axis of diffusion to proceed from one area to another along the fiber structures anatomically connecting the two. Many variants of this line propagation technique have been proposed (Basser et al., 2000; Conturo et al., 1999; Jones et al., 1999; Lazar et al., 2003; Lori et al., 2002; Mori et al., 1999; Poupon et al., 2000; Westin et al., 2002; Xue et al., 1999), and many of the large white matter structures such as the cortical association tracts can be reliably traced in individual subjects using these methods (Catani et al., 2002; Mori et al., 2002; Wakana et al., 2004).

However, DTI tractography is known to have limitations primarily resulting from partial volume averaging of complex fiber architecture (such as sharp curves and crossings) into single voxels (Alexander et al., 2001; Tuch et al., 2002, 2003). Since typical voxel sizes for in vivo human DTI are in the range of $2 \mathrm{~mm}$ (i.e. $8 \mathrm{~mm}^{3}$ ), some white matter voxels can easily contain multiple fiber populations with different directions. The diffusion tensor (DT) model is a 3D Gaussian profile constrained to a single maximum. When two fiber bundles cross at (nearly) $90^{\circ}$, the tensor will take an oblate (planar) shape in the plane of crossing. When bundles cross at less than $90^{\circ}$ the tensor will have a prolate shape with its primary axes in between those of the directions of the crossing bundles. In 
either case, the estimated tensor model misrepresents the actual diffusion profile. Adding to the problem, the DT model will also be unable to distinguish a crossing $(\mathrm{X})$ from a kissing $(><)$ configuration since both give rise to the same actual diffusion profile. Another situation that cannot be accurately represented by the DT model is when a single tract curves sharply within a single voxel. This would lead to a continuous range of directions present in a single voxel, a non-Gaussian diffusion profile which the DT model is unable to represent since it assumes the presence of a single coherent direction. An estimated DT model for such a voxel will quickly degrade towards an oblate or spherical shape which only represents the average direction of the curve at a low modeled anisotropy.

Furthermore, some inherent limitations exist in deriving with certainty the existence or absence of anatomical connections between brain regions from tractography results. Line propagation algorithms will often stop at crossings or high curvature regions, either because the estimated anisotropy does not exceed a fixed threshold or because of the often inbuilt bias against high curvature tracts in the form of the angle threshold. Additionally, situations of convergence (multiple bundles merge into one) and/or divergence (a bundle splits into two) also yield considerable trouble for DT tractography approaches. At a voxel where convergence takes place, information is lost about which fibers merged into the main tract and which were present already. Similarly, at the site of divergence it is unclear which axon bundles leave the main tract and which continue along it.

Some of the inherent limitations of the diffusion tensor model and line propagation algorithms may be mitigated by an increase in spatial acquisition resolution. If converging and diverging bundles maintain a coherent location within the main tract as they follow it along, an increase in spatial resolution may resolve some of the ambiguity. The idea is that if the main tract is sampled densely enough, the diverging or converging parts might be kept separate as the tract continues its course. Problems with high curvature tracts can also be mitigated by an increase in spatial resolution. Diffusion tensors become more planar with less well defined principle direction when the voxel size increases much beyond the radius of curvature of a tract. With a resolution increase to a voxel size smaller than the radius of tract curvature, the locally estimated principle diffusion directions will become sharper defined. Potentially, increased spatial resolution can resolve some kissing vs. crossing situations in a similar way. If the curvature of the tracts at the kissing point is low enough with respect to the voxel size and the 'kiss' is not too close, each might be followed through without erroneously crossing over.

In this study, high-resolution (156 $\mu \mathrm{m}$ in-plane) diffusion tensor imaging of the human optic chiasm was performed ex vivo at ultrahigh field $(9.4 \mathrm{~T})$. Water diffusion anisotropy has been shown to be preserved in formalin-fixed tissue (Guilfoyle et al., 2003; Sun et al., 2005, 2003). This makes the post-mortem investigation of fixed tissue samples with DTI at high field strengths an attractive candidate for the evaluation of the diffusion tensor model and tractography algorithms and the effects of spatial resolution on their performance. The optic chiasm, with its complex microstructure of fibers, which kiss, cross, converge and curve, is a challenge for the capabilities of the diffusion tensor model and tractography methods. Since the macroscopic connectivity through the optic chiasm is well known it is an ideal candidate for evaluation of DTI tractography approaches in resolving inter-regional connectivity.
We briefly review the pathways of the optical projections through the optic chiasm in the human (see Standring, 2005). The optic nerves, arising from the ganglion cells of the retina, project in largest part to the lateral geniculate nucleus (LGN) in the thalamus. From the LGN, the optic radiation projects to the primary visual cortex in the calcarine fissure of the occipital lobe (see Fig. 1). A retinotopic organization is kept throughout the projections from the eye to the occipital cortex. The optic nerves pass dorsally into the brain, ventral to the orbitofrontal cortex, to meet at the midline in the optic chiasm, an oblate structure where the optic fibers partially decussate and merge into the optic tracts. Optic fibers carrying input from the temporal half of the retina (including the temporal half of the fovea), representing the contralateral half of the visual field, pass into the ipsilateral optic tract in the optic chiasm. Fibers arising from the nasal hemiretinae, representing the ipsilateral visual hemifield, decussate in the optic chiasm to enter the contralateral optic tract. Decussating fibers do not cross the chiasm in an exclusively diagonally oriented path. Rather, they curve sharply to cross the chiasm in a medio-lateral direction then curve sharply again to align with the temporal fibers of contralateral optic nerve and enter into the contralateral optic tract (Standring, 2005). Such highly curved paths are a problem for most fiber tracking algorithms that (implicitly) assume fibers to be guided by shortest path and minimum curvature constraints. Decussating fibers have even been reported to loop anteriorly into the contralateral optic nerve, before curving sharply again (in what is known as 'Wilbrand's Knee') and enter into the optic tract (Wilbrand, 1926). However, the existence of Wilbrand's Knee in the normal human optic chiasm is disputed (Horton, 1997; Lee et al., 2006).

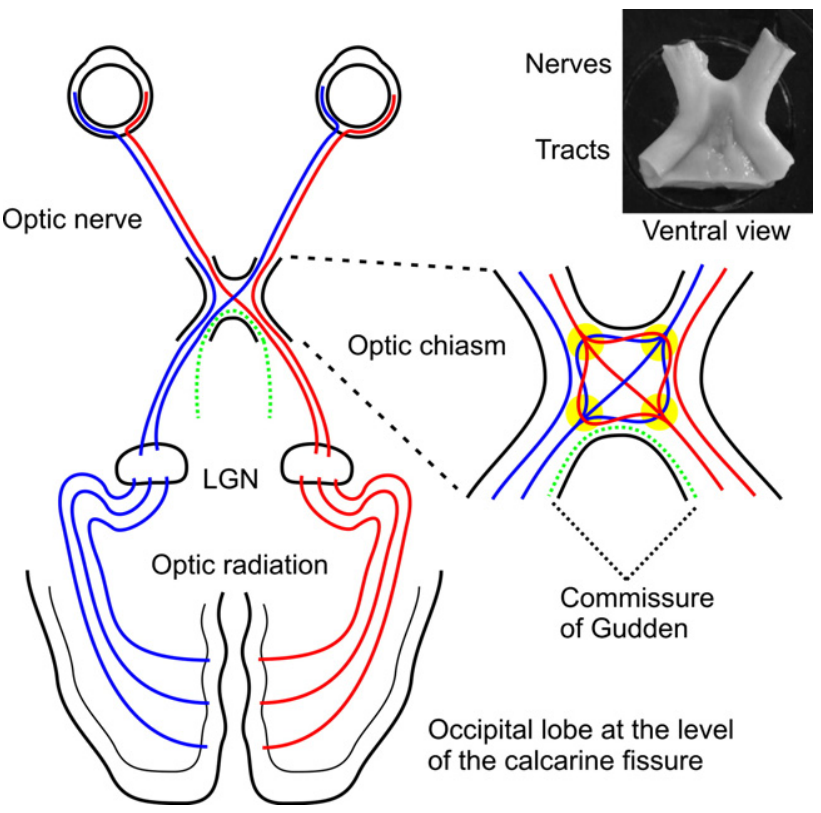

Fig. 1. The anatomy of the human optic pathways and optic chiasm. Red lines are nerves representing the left visual hemifield, blue lines are nerves representing the right visual hemifield. Yellow circles mark regions of high curvature, crossings, divergence and convergence, problematic for the diffusion tensor model and line propagation algorithms. LGN: lateral geniculate nucleus. The inset in the upper right corner shows one of the tissue samples (OC3), photographed from the ventral aspect. Remains of the pineal gland and hypothalamus can be seen in the optic tracts. 
The majority of all optic nerve fibers cross in the chiasm and about two thirds of the central chiasm is occupied by fibers arising from the foveal region of the retinae, passing mostly through the dorsal part of the central chiasm. The fibers arising from the extrafoveal part of the nasal hemi-retinae decussate more dorsally in the optic chiasm. The ventral-most portion of the central chiasm is crossed by fibers representing the lateral monocular part of the visual hemifields (Standring, 2005). The posterior and medial parts of the optic chiasm are thought to contain fibers that are not derived from the optic nerves. These fibers form the ventral supra-optic commissure or commissure of Gudden, possibly connecting the medial geniculate nuclei of the two sides. Gudden's commissure is one of the three supra-optic commissures, which also include the dorsal supra-optic commissure (commissure of Meynert) and the anterior supra-optic commissure (commissure of Ganser). The connections of the supra-optic commissures in humans are uncertain, and only the dorsal and ventral commissures are recognized in most anatomical nomenclature.

\section{Methods}

\section{Tissue preparation}

Tissue probes containing the optic chiasms were obtained from the brains of four subjects (two female, 23 and 33 years old at death; two male, 26 and 34 years old at death, free of known neurological disorders) during routine autopsy examination. The procedures had been approved by the ethical committee of the University Clinic, Frankfurt. After a postmortem delay of 9 to $20 \mathrm{~h}$, the optical chiasm along with proximal aspects of the optic nerves and optic tracts (and in two cases parts of the hypothalamus) was prepared and fixed for $48 \mathrm{~h}$ in a solution containing $2.6 \%$ paraformaldehyde (PFA), $0.8 \%$ iodacetic acid, $0.8 \%$ sodiumperiodate, and $0.1 \mathrm{M}$ D-L-lysine in $0.1 \mathrm{M}$ phosphate buffer at $\mathrm{pH}$ 7.4. Subsequently, the tissue was washed in phosphate buffer 1 week prior to scanning and stored in $2 \%$ PFA in phosphate buffer at $\mathrm{pH} 7.4$ at $37{ }^{\circ} \mathrm{C}$. The time between the start of fixation and MR scanning of the tissue ranged from 26 to 66 days. The tissue samples were embedded in $2.5 \%$ agar in $0.9 \%$ $\mathrm{NaCl}$ solution for MR scanning.

\section{MR imaging}

Experiments were performed with a $9.4 \mathrm{~T} / 31 \mathrm{~cm}$ horizontal MRI scanner (Magnex Scientific, UK) equipped with $30 \mathrm{G} / \mathrm{cm}$ gradients $(11 \mathrm{~cm} \mathrm{ID,} 300 \mu$ s rise time, Magnex Scientific) and driven by a

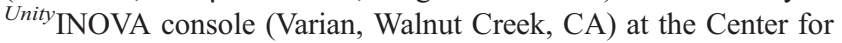
Magnetic Resonance Research (CMRR), University of Minnesota Medical Center. The RF coil was a home-built quadrature surface coil, composed of two $3.3 \mathrm{~cm}$ diameter partially overlapping coils. Diffusion weighted MR images were acquired using a multi-shot pseudo-3D double spin-echo echo-planar imaging (SE-EPI) sequence with two phase encoding directions and four segments per pseudo-slice. The following parameters were used: TR/TE= $2000 \mathrm{~ms} / 35 \mathrm{~ms},|g|=17$ gauss $/ \mathrm{cm}, \delta=6 \mathrm{~ms}, \Delta=15 \mathrm{~ms} \quad(b=$ $1584 \mathrm{~s} / \mathrm{mm}^{2}$ for each direction). Six gradient combinations according to the direction scheme $(X, Y, 0),(X, 0, Z),(0, Y, Z)$, $(X,-Y, 0),(0, Y,-Z),(-X, 0, Z)$ and one image with minimal gradient strength on three directions was acquired as the un-weighted image. The field of view (FOV) was $2 \times 2 \times 2 \mathrm{~cm}^{3}$, the matrix size was $128 \times 128 \times 64$ (giving a nominal resolution of $156 \times 156 \mu \mathrm{m}$ in-plane with a slice thickness of $312 \mu \mathrm{m}$ ). Shimming for field inhomogene- ities was done using FASTMAP to include high order shims in an analytical manner. Since the sample was in a cylindrical container, homogeneously filled with agar gel, whose magnetic susceptibility is extremely similar to that of the tissue sample, shimming was close to perfect. The water resonance had no broad components or strong deviations from Lorenzian, meaning that within the shim volume the line shape was approximately the natural line shape of agar gel/tissue. Moreover, since the acquisition was done with multi-shot EPI, the effective bandwidth on the phase encoding direction was approximately $5000 \mathrm{~Hz}$. With a line width of approximately $10 \mathrm{~Hz}$, possible geometric distortions due to field inhomogeneity are on the order of fractions of a pixel. A total of 64 repetitions for each direction were acquired and averaged. No spatial transformations were applied to the imaging volumes. One dataset had to be discarded due to inferior data quality. Time to fixation was shortest for this sample $(9 \mathrm{~h})$, but time between fixation and scanning was longest (66 days), though not significantly longer than some of the other samples (e.g. second longest time between fixation and scanning was 59 days). Estimation of diffusion tensors and tractography was performed on the remaining three optic chiasm datasets (OC1, OC2 and OC3).

\section{Estimation of diffusion tensors}

All processing and visualization were performed in custom written $\mathrm{C}++$ software, using the OpenGL graphics library (www. opengl.org) for 3D visualization. Within each voxel, the local diffusion profile can be captured in a 3-dimensional rank-2 tensor: the diffusion tensor (Basser et al., 1994) that can be geometrically represented as an ellipsoid. The diffusion tensor (DT) models the diffusion profile in a voxel as a 3D Gaussian with three orthogonal eigenvectors or axes of diffusion (the primary or major eigenvector $\mathbf{e}_{1}$, the secondary or median eigenvector $\mathbf{e}_{2}$ and the tertiary or minor eigenvector $\mathbf{e}_{3}$ ) and their corresponding eigenvalues or diffusion constants $\lambda_{1}, \lambda_{2}$ and $\lambda_{3}$. The diffusion tensor models the signal $S$ for a diffusionweighting direction $\mathbf{g}=\left(g_{x}, g_{y}, g_{z}\right)$ with respect to a non-diffusion weighted image $S_{0}$ as:

$S / S_{0}=e^{b \mathbf{g}^{T} \mathbf{D g}}$

where $b$ is the $b$-value, quantifying the strength of diffusion weighting. After taking the natural logarithm on both sides of Eq. (1), the set of acquired diffusion gradient directions defines a linear system of equations, solvable for the tensor components $D_{i j}$ (Basser et al., 1994). This system of equations was solved in the least-squares sense by a singular value decomposition (Press et al., 2002) to estimate the diffusion tensor model voxel-wise. Each tensor was then diagonalized by Jacobi rotations (Press et al., 2002) to obtain the three eigenvectors and corresponding eigenvalues. Eigenvectors were sorted with respect to the magnitude of the corresponding eigenvalues to get the primary, secondary and tertiary axes of diffusion $\mathbf{e}_{1}, \mathbf{e}_{2}$ and $\mathbf{e}_{3}$, and their corresponding diffusion constants $\lambda_{1} \lambda_{2}$ and $\lambda_{3}$, where $\lambda_{1}>\lambda_{2}$ $\lambda_{3}$. Estimated tensors are visualized as cylinders in the direction of the primary axis of diffusion $\mathbf{e}_{1}$ with length proportional to $\lambda_{1}$, and as 'boxoids', rectangular 3-dimensional boxes with axes proportional to $\lambda_{1} \mathbf{e}_{1}, \lambda_{2} \mathbf{e}_{2}$ and $\lambda_{3} \mathbf{e}_{3}$. Both cylinders and boxoids are color-coded for direction of the primary axis of diffusion via the standard directional coding scheme with left to right directions 
colored red, anterior to posterior directions colored green and inferior to superior directions colored blue (Pajevic and Pierpaoli, 1999).

Various scalar quantities were derived from the eigenvalues $\lambda_{1}$, $\lambda_{2}$, and $\lambda_{3}$ of the estimated diffusion tensors. Mean diffusivity is given by

$\mathrm{MD}=\frac{\text { Trace } /(D)}{3}=\frac{\lambda_{1}+\lambda_{2}+\lambda_{3}}{3}$

which is proportional to the volume of the ellipsoid and quantifies the absolute amount of diffusion in a voxel. Fractional anisotropy (Pierpaoli and Basser, 1996), given by

$\mathrm{FA}=\frac{\sqrt{3\left(\lambda_{1}-\bar{\lambda}\right)^{2}+3\left(\lambda_{2}-\bar{\lambda}\right)^{2}+3\left(\lambda_{3}-\bar{\lambda}\right)^{2}}}{\sqrt{2\left(\lambda_{1}^{2}+\lambda_{2}^{2}+\lambda_{3}^{2}\right)}}, \bar{\lambda}=\frac{\lambda_{1}+\lambda_{2}+\lambda_{3}}{3}$

quantifies the amount of variance present in the three eigenvalues of the diffusion tensor and is high where tensors have a highly linear or prolate shape, with the primary eigenvalue much larger than the other two, indicating the presence of a single highly coherent fiber bundle. Although FA is highly suitable to quantify the absolute amount of anisotropy (effectively the deviation from a spherical shape), it is not able to distinguish well between linear and planar tensor shapes. Geometric measures of diffusion have been proposed that are better suited to discern linear from planar and spherical tensor shapes (Westin et al., 2002):

$C_{1}=\frac{\lambda_{1}-\lambda_{2}}{\lambda_{1}}, C_{\mathrm{p}}=\frac{\lambda_{2}-\lambda_{3}}{\lambda_{1}}, C_{\mathrm{s}}=\frac{\lambda_{3}}{\lambda_{1}}, C_{1}+C_{\mathrm{p}}+C_{\mathrm{s}}=1$

The linear measure $C_{1}$ is high for linear or prolate tensor shapes, where $\lambda_{1} \gg \lambda_{2} \approx \lambda_{3}$. The planar measure $C_{\mathrm{p}}$ is high for planar or oblate tensor shapes, where $\lambda_{1} \approx \lambda_{2} \gg \lambda_{3}$. The spherical measure $C_{\mathrm{s}}$ is high for spherical tensor shapes, where $\lambda_{1} \approx \lambda_{2} \approx \lambda_{3}$. A quantity similar in behavior to $\mathrm{FA}$ is $1-C_{\mathrm{s}}$, quantifying the deviation from a spherical shape, without distinguishing between linear and planar shapes. A quantity which is better able to identify non-linear tensor shapes is $1-C_{1}$, quantifying the deviation from a linear shape. Maps of the $C_{1}$ coefficient were used to highlight problematic regions of crossing, kissing, convergence and high curvature, with the expectancy that these regions will show a reduction in the estimated $C_{1}$ coefficient.

\section{DT tractography}

Constructing a continuous tract line $\mathbf{r}(s)=(x(s), y(s), z(s))$ from a tensor field $\mathbf{D}(x, y, z)$ can be formulated as the solution of an ordinary differential equation (Basser et al., 2000):

$\frac{\mathrm{d} \mathbf{r}(s)}{\mathrm{d} \mathbf{s}}=f(\mathbf{D}(\mathbf{r}(s)))$

where $\mathbf{r}(s)$ is parameterized by position $s$ along the tract, $\mathbf{D}(\mathbf{r}(s))$ denotes the tensor at position $\mathbf{r}(s)$ for a given $s$, and $f$ denotes a function giving the tangent to $\mathbf{r}(s)$ from the local tensor at $\mathbf{r}(s)$. In practice a discrete tract-line $\mathbf{r}(i)=\left(x_{i}, y_{i}, z_{i}\right)$, with $i=0, \ldots, N$, will have to be constructed from a discrete estimated tensor volume $\mathbf{D}(i, j, k)$. Line propagation or streamline approaches to fiber tracking trace a tract-line through a tensor volume by following the local estimated diffusion directions from a seedpoint. The seedpoint $\left(x_{0}, y_{0}, z_{0}\right)$ forms the boundary condition for the numerical process of solving a discretized differential equation. In its simplest form a line propagation algorithm amounts to approximating a solution by Euler's method:

$\mathbf{r}(i+1)=\mathbf{r}(i)+\alpha^{*} \mathbf{v}(i), \mathbf{v}(i)=f(\mathbf{D}(\mathbf{r}(i)), \mathbf{v}(i-1))$

Starting from the seedpoint a step of stepsize $\alpha$ is taken in a direction $\mathbf{v}(i)$ determined by the local tensor $\mathbf{D}$ and the previous tracking direction $\mathbf{v}(i-1)$. The simplest form of direction determination would be to set $\mathbf{v}(i)=\mathbf{e}_{1}(\mathbf{r}(i))$, i.e. to use the primary eigenvector of the local tensor as the new tracking direction. The Euler method as an ODE solver is known to be unstable and inaccurate when stepsizes are not extremely small. Better methods exist that use information at multiple points near the step-location to yield better stability and accuracy, such as the fourth order Runge-Kutta method (Press et al., 2002) that was used here, with a stepsize of $50 \mu \mathrm{m}$.

Taking the primary eigenvector of the local tensor as the new tracking direction can work well in regions where a single coherent tract is present. However, this strategy can lead to errors when more complex configurations, such as crossings, are encountered. As discussed above, the primary eigenvector often does not represent a veridical tract direction in the case of a crossing. An approach to direction determination that uses the full local tensor information can perform better in this case. It has been proposed to use the local tensor $\mathbf{D}$ as a projection operator to deflect the previous tracking direction towards the directions compatible with the local tensor (Lazar et al., 2003; Westin et al., 2002) by defining $f$ as:

$\mathbf{v}(i)=f(\mathbf{D}(\mathbf{r}(i)), \mathbf{v}(i-1))=\mathbf{D}(\mathbf{r}(i)) * \mathbf{v}(i-1)$

The new tracking direction is formed as the matrix-vector product of the local tensor and the old tracking direction. In cases of fiber crossing, the inertia of the old direction will lead this operation to trace through the planar tensor at the crossing site, when using only the primary eigenvector might fail. It should be noted that this inertial bias might also lead to a wrong interpretation of kissing sites or high curvature bends as crossings where a tract continues a relatively straight path. To avoid noise contributions from eigenvectors with small eigenvalues, only the larger eigenvectors can be made to contribute to the projection operation. The full tensor can be written in terms of its eigenvector and eigenvalues:

$\mathbf{D}=\lambda_{1} \mathbf{e}_{1} \mathbf{e}_{1}^{T}+\lambda_{2} \mathbf{e}_{2} \mathbf{e}_{2}^{T}+\lambda_{3} \mathbf{e}_{3} \mathbf{e}_{3}^{T}$

This sum can be truncated to include only the terms that are considered to be large enough. A possible truncation strategy is that of Westin et al. (2002), who propose to select the contributing eigenvectors from the relative magnitudes of the $C_{\mathrm{l}}, C_{\mathrm{p}}$, and $C_{\mathrm{s}}$ coefficients. When $C_{1}$ is largest, only $\mathbf{e}_{1}$ is used, when $C_{\mathrm{p}}$ is largest $\mathbf{e}_{1}$ and $\mathbf{e}_{2}$ are used, and the full tensor is used when $C_{\mathrm{s}}$ is largest.

Fiber tracking was performed by a line propagation algorithm using the truncated tensor projection operator described above for local direction determination. The local tensor $\mathbf{D}$ at an arbitrary position $\mathbf{r}(s)$ in the discrete volume $\mathbf{D}(i, j, k)$ was computed by trilinear interpolation of the individual tensor elements. The resulting interpolated tensor was then re-diagonalized by Jacobi rotations. Tracing was stopped either when the local FA was below 0.15 or when the angle between previous and current tracking direction was larger than $120^{\circ}$. The very liberal angle threshold was used because 
high curvature bends were to be expected from the known anatomy of the optic chiasm. Regions of interest (ROIs) were drawn as contiguous patches of voxels on coronal or sagittal slices through the left and right optic nerves and tracts of each chiasm. Seedpoints for the tractography were placed in a $3 \times 3 \times 3$ grid within each seed voxel in an ROI. Line propagation tractography was initiated for each seedpoint, both in the positive and negative direction of the local primary eigenvector. Tracked fibers from the resulting set for each ROI were then post-selected for inclusion in each of the other three ROIs to obtain fiber sets with given ROIs as initiation and termination sites. Effectively this amounts to an 'AND' operation for inclusion in both ROIs (Wakana et al., 2004).

\section{Spatial down-sampling}

To investigate the effects of spatial resolution on the characterization of diffusion and tractography results, down-sampled versions of OC3 were created. The down-sampled versions were produced by averaging contiguous blocks of voxels in the original dataset into larger voxels. Three down-sampled versions of the OC3 dataset were created, averaging blocks of $1 \times 2 \times 2,2 \times 4 \times 4$ and $4 \times 8 \times 8$ voxels, respectively, where the first dimension is the (pseudo-)slice dimension. This resulted in datasets with isotropic voxel resolutions of $312.5 \mu \mathrm{m}, 625 \mu \mathrm{m}$ and $1250 \mu \mathrm{m}$, respectively. Diffusion tensors were estimated, ROIs were drawn, line propagation tracking was initiated, and tracked streamlines were postselected in each of these datasets as in the original dataset. The seedpoint grid for tractography in each voxel in the down-sampled datasets was increased in density to $3 \times 4 \times 4,3 \times 5 \times 5$ and $4 \times 8 \times 8$, respectively, to obtain a similar seedpoint density to the original trackings.

\section{Results}

Fig. 2 shows maps of $C_{1}$ in near-axial slices for OC3. As argued above, regions of low $C_{1}$ should be characterized by non-prolate estimated tensor shapes, indicative of curvature, crossing and/or convergence. OC3 indeed shows clear zones of highly reduced $C_{1}$, at the lateral-most aspects of the central chiasm where it intersects with the medial aspects of the optic nerves and tracts. The enlarged detail renderings show the estimated primary directions of diffusion to change rapidly over a very short distance in these zones, and the boxoid rendering indicates predominantly planar tensor shapes there. Visual inspection of primary eigenvector directions suggests evidence of sharp curves (e.g. Fig. 2A, third panel), crossings (Fig. 2B, third panel) and convergence and divergence (e.g. Fig. 2B,
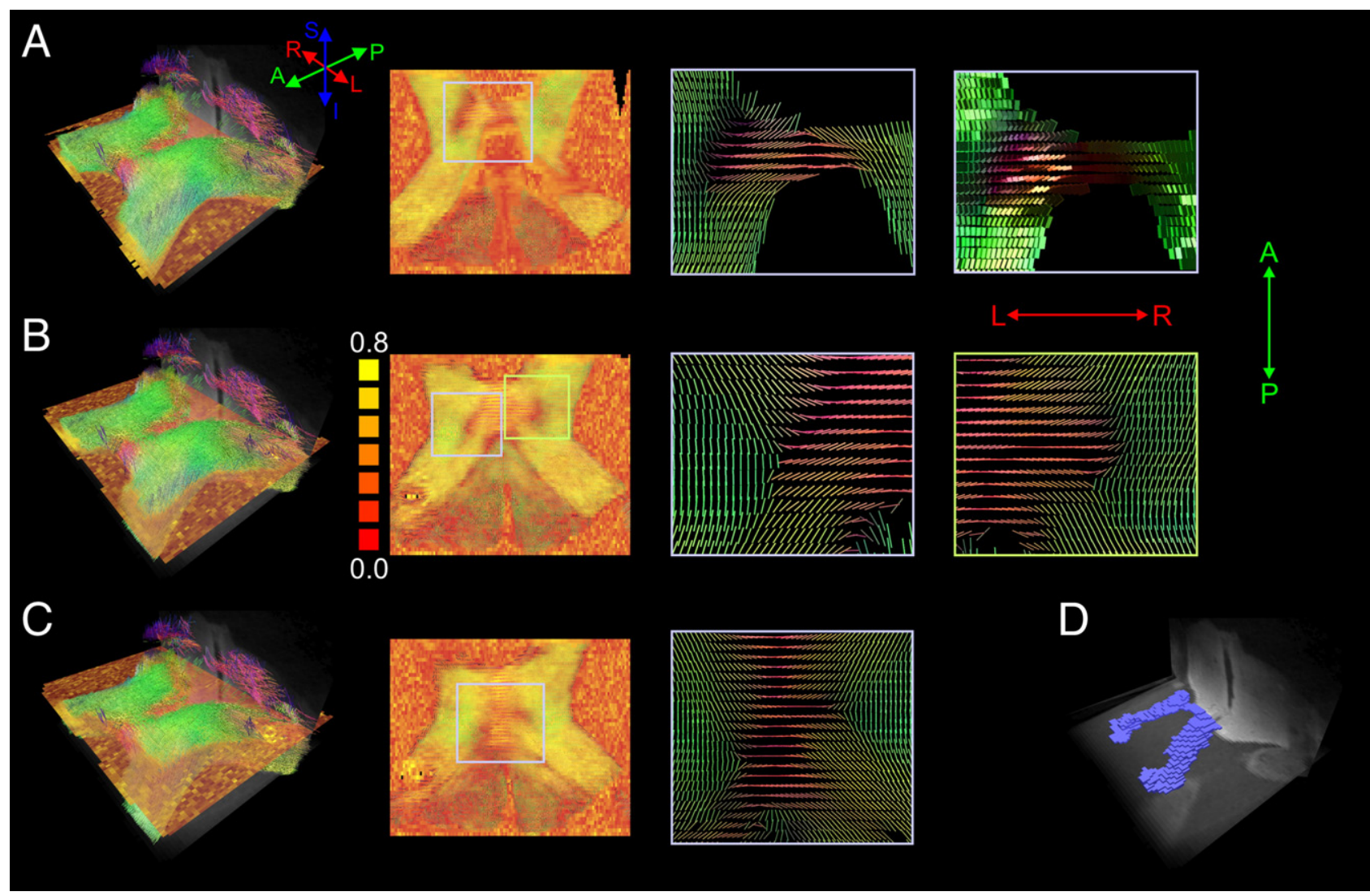

Fig. 2. (A-C) Near-axial slice planes through OC3 at different levels. Left column shows a 3D volume rendering of the chiasm color-coded for the local direction of largest diffusion (see inset) and showing the axial slice positions for A, B and C. Color coding in the second column represents the linear shape measure from $C_{1}=0$ (red) to $C_{1}=0.8$ (yellow). Overlaid on the slices are the local axes of largest diffusion, rendered as cylinders. Enlarged regions of the slices show cylinder renderings of the largest axis of diffusion and a boxoid rendering (top row) representing all three axes of the local diffusion tensor. Both cylinders and boxoids are color-coded for direction of the primary axes of diffusion. (D) A 3D rendering of the zones of low $C_{1}$, indicating the presence of non-prolate tensor shapes due to curvature, crossing, convergence and/or divergence of tracts. 
fourth panel). Chiasms OC1 and OC2 showed very similar patterns of tensor shapes and directions. Fig. 2D shows a 3D rendering of the zones of low $C_{1}$ for OC3. Interestingly, the pattern is not one of four discrete spots of high curvature and crossing (or 'pillars' in 3D), as suggested by the yellow highlights in Fig. 1, but two zones that proceed bilaterally from the anterior and ventral aspect to the posterior and dorsal aspect of the lateral central chiasm, forming diagonal bands. Chiasms OC1 and OC2 also showed this spatial pattern of zones or reduced $C_{1}$.

The estimated tensors for the crossing fibers in the central chiasm (see Fig. 2B, third and fourth panel, but also Fig. 4) are of particular interest. Fibers from the medial aspect of the optic nerves each pass over to the other side of midline there and cross each other in the process. The estimated primary directions of diffusion suggest that this crossing is mostly one of a very low angle, caused by the nerves curving into a near left-right direction before passing the midline. However, the pure left-right orientation of primary eigenvector at the midline is likely to be misleading. As discussed above, the tensor model is not able to accurately represent the non-Gaussian multipeaked diffusion profile of a fiber crossing. Most likely the average direction of the two crossing fibers is what is represented at the crossing sites in the central chiasms.
Selected streamlines resulting from the fiber trackings are shown in Fig. 3. Absolute numbers and relative percentages of tracked fibers with a given seeding ROI and termination area are listed in Table 1. From the known anatomy of the chiasm, the majority of fibers tracked from the seed ROIs in the left and right optic nerves are expected to pass through the contralateral optic tract (about $75 \%$ ). The remaining minority of streamlines should pass through the ipsilateral tract. There are no known axonal connections between the eyes through the optic chiasm, so fibers should not be tracked between the ROIs in the optic nerves. Nearly the same expectations hold (mutatis mutandis) for fibers tracked from the ROIs in the optic tracts. The majority should pass through the contralateral optic nerve and a minority through the ipsilateral nerve. Connections between the two optic tracts are known to exist (see Fig. 1), although these are expected to be even less numerous.

Fig. 3 and Table 1 show that almost all connections between ROIs are tracked (including the non-veridical connections between optic nerves) though in varying relative proportions. On the whole symmetry exists in the amount of fibers tracked in either direction between two ROIs (i.e. a similar amount of fibers are tracked from A to $\mathrm{B}$ and from $\mathrm{B}$ to $\mathrm{A})$. Fibers crossing from optic nerve to contralateral optic tract (or vice versa), which should be the most
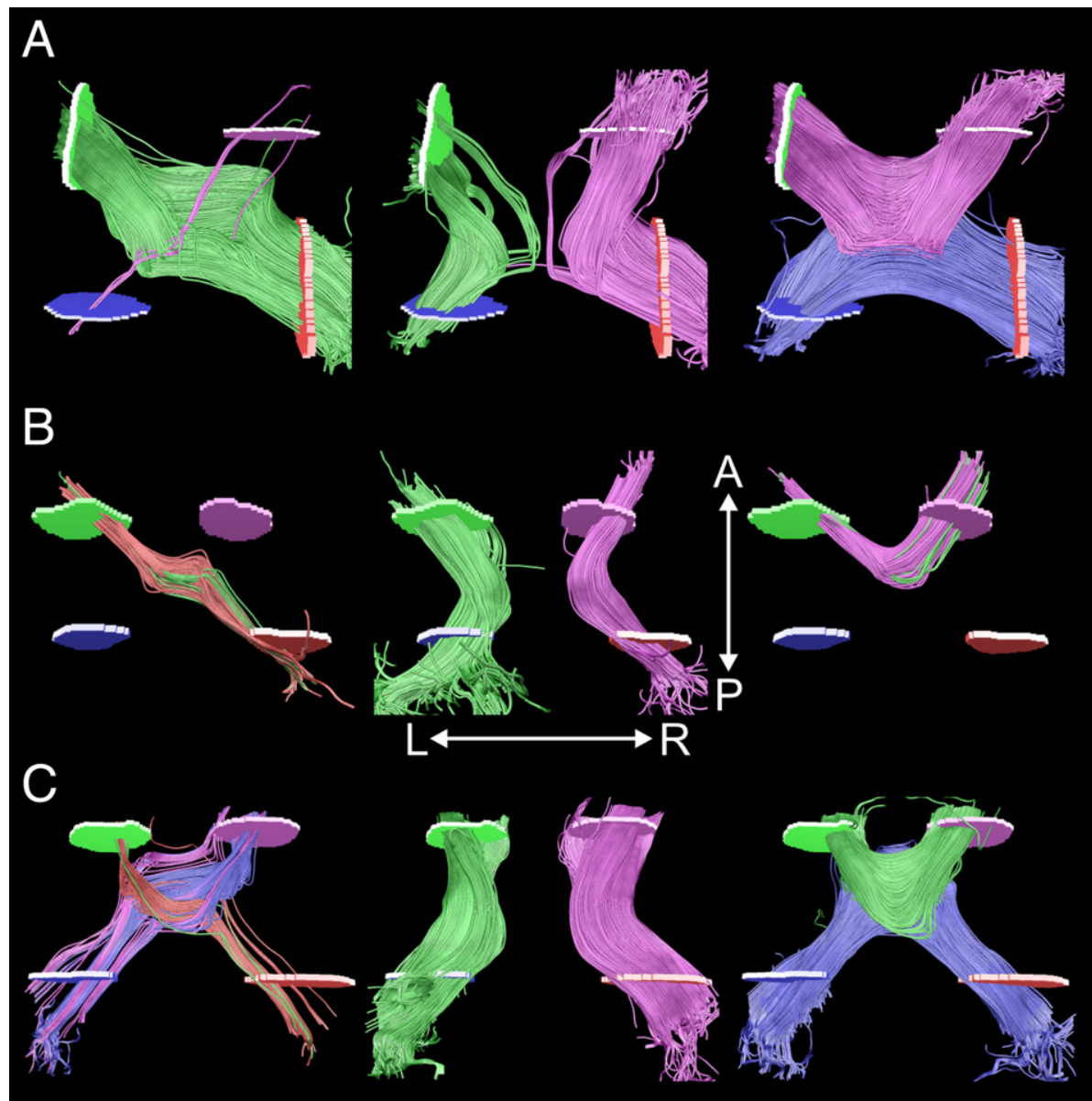

Fig. 3. Selected fiber tracking results for OC1 in A (top row), OC2 in B (middle row), and OC3 in C (bottom row). Seed regions are shown for the left optic nerve (in green), the right optic nerve (in purple), the left optic tract (in blue), and the right optic tract (in red). All displayed tracking results are colored according to the seed region from which they were initiated. Post-selection for passage through a second ROI (effectively an AND operation) was performed to group tractography results with the same start and end regions. Left column shows streamlines crossing both the horizontal and vertical midline, middle column shows streamlines connecting optic nerve and optic tract on the same side, right column shows streamlines connecting either optic nerves or optic tracts on both sides. 
Table 1

Absolute numbers and percentages (in brackets) of streamlines tracked from a given seed location (row) that passes through a given target location (column) for all three OCs

\begin{tabular}{|c|c|c|c|c|c|}
\hline & & Left nerve & Right nerve & Left tract & Right tract \\
\hline \multicolumn{6}{|l|}{$O C 1$} \\
\hline Left nerve & $(6752)$ & - & $2553(50 \%)$ & $1268(25 \%)$ & $1239(25 \%)$ \\
\hline Right nerve & (3159) & $1222(58 \%)$ & - & $6(0.3 \%)$ & $893(42 \%)$ \\
\hline Left tract & (2864) & $488(27 \%)$ & $0(0 \%)$ & - & $1322(73 \%)$ \\
\hline Right tract & $(7252)$ & $1384(32 \%)$ & $812(19 \%)$ & $2121(49 \%)$ & - \\
\hline \multicolumn{6}{|l|}{$O C 2$} \\
\hline Left nerve & (3198) & - & $142(9 \%)$ & $1412(87 \%)$ & $64(4 \%)$ \\
\hline Right nerve & (2613) & $452(37 \%)$ & - & $0(0 \%)$ & $767(63 \%)$ \\
\hline Left tract & (3133) & $1387(100 \%)$ & $0(0 \%)$ & - & $0(0 \%)$ \\
\hline Right tract & (3332) & $235(14 \%)$ & $1504(86 \%)$ & $0(0 \%)$ & - \\
\hline \multicolumn{6}{|l|}{$O C 3$} \\
\hline Left nerve & (7141) & - & $1300(36 \%)$ & $2114(59 \%)$ & $182(5 \%)$ \\
\hline Right nerve & $(6752)$ & $1373(37 \%)$ & - & $166(5 \%)$ & $2135(58 \%)$ \\
\hline Left tract & (7814) & $4738(75 \%)$ & $287(5 \%)$ & - & $1322(20 \%)$ \\
\hline Right tract & (8690) & $147(2 \%)$ & $5029(81 \%)$ & $1054(17 \%)$ & - \\
\hline
\end{tabular}

Percentages are relative to all streamlines that reached a location (i.e. relative to the sum of streamlines in a row). The absolute number of lines tracked from the seed location (longer than $2 \mathrm{~mm}$ ) is listed in brackets.

numerous, are found the least. Whereas the proportion of all fibers tracked from a ROI in the optic nerve or tract should be around $75 \%$, it is only found to be in the range of $0-10 \%$ for OC2 and OC3. For OC1, it is about $30 \%$, but only for connections between left nerve and right tract (see Fig. 3, left column). Connections between optic nerve and ipsilateral optic tract, expected in proportions of about $25 \%$, are overrepresented with proportions of tracked fibers between $19 \%$ and $42 \%$ for OC $1,63 \%$ and $100 \%$ for OC 2 and $58 \%$ and $81 \%$ for OC3 (see Fig. 3, middle column). Connections between the optic nerves that should not exist are tracked in considerable numbers with proportions of $9 \%$ to $58 \%$ of all fibers tracked from the optic nerve ROIs (see Fig. 3, right column). Finally, the proportion of fibers that run through both the optic tracts, connecting the optic tract ROIs, also seem to be overrepresented in OC1 at $49 \%$ to $73 \%$ and OC3 at $17 \%$ to $20 \%$ but are completely absent in OC2. Overall, fibers crossing from optic nerve to contralateral optic tract, which should be the most numerous, are underrepresented, whereas fibers connecting ROIs on the same side of the horizontal or vertical midline are heavily overrepresented.

Detail renderings of selected fibers of the above classes where they pass the critical high curvature regions, overlaid on primary eigenvector renderings, are very informative in clarifying the pattern of connectivity described above. Fig. 4A shows selected trackings of both the left and the right optic nerve ROI for OC1. The selected streamlines of the left optic nerve ROI pass through either the left or right optic tract ROIs. Although these connections are likely to reflect existing fibers, the path traced by these tracked streamlines is unlikely to be veridical. The tracings terminating in the contralateral optic tract were initiated in the temporal part of the optic nerve where fibers are situated that generally pass to the ipsilateral optic tract. The tracing terminating in the ipsilateral tract was initiated in the nasal part of the left optic nerve from where fibers generally cross to the contralateral optic tract. These seemingly non-veridical tracings seem to arise from the problematic region enlarged in Fig. 4A, where (most likely) fibers passing from the left optic nerve to the left and right optic tracts diverge, and fibers from the right optic nerve en route to the left optic tract converge with left optic nerve fibers with the same target. Furthermore, slightly left (medial) of this 'three-fold kissing' the left and right decussating nerve tracts cross each other (see inset at the top right of Fig. 4A). A similar critical branching point should exist on the other side of the midline. The selected streamlines originating from the right optic nerve ROI pass through either the left optic nerve ROI or the right optic tract ROI, neither of which is likely to be a veridical tract. The 'eye-to-eye' connection in particular seems to be the result of a wrong turn made at both the left and right critical branching points.

Fig. 4B shows a detail of selected trackings for OC2 initiated from the medial part of the left optic nerve ROI. Two sets of streamlines are depicted that initially follow a very similar path posteriorly through the optic nerve and then curving medially into the central chiasm. However, at the branch point on the contralateral side of the central chiasm the tracked bundle splits into one group of tracts that turns posteriorly into the right optic tract and a second group that turns anteriorly into the right optic nerve. It can be seen that the turn at the branching point is determined by the relative spatial position and the precise angle at which it is entered, owing to the purely local information used by the tracking algorithm and the slight inertia exercised by the previous tracking direction.

Fig. $4 \mathrm{C}$ shows a detail rendering of streamlines originating in the left and right optic tract ROIs of OC3. It shows seemingly veridical trackings between the optic nerves and contralateral tracts and incorrect tracked fibers connecting the left and right optic nerve. The latter are seen to arise from the left-to-right orientation of the primary eigenvectors at the midline of the central chiasm where left and right optic nerve projections cross, as discussed above. Instead of continuing their course posteriorly across the midline, the left optic nerve streamlines are deflected to a path straight across the midline and then erroneously follow the right optic nerve projections retrogradely toward the right eye. The tracked fibers between the optic nerves and contralateral tracts curve similarly towards a rightleft orientation near the midline but, importantly, maintain a slightly diagonal anterior-posterior course, curving towards the anteriorposterior direction again on the other side of the midline. Note also that these two streamline groups cross each other (slightly offset in 


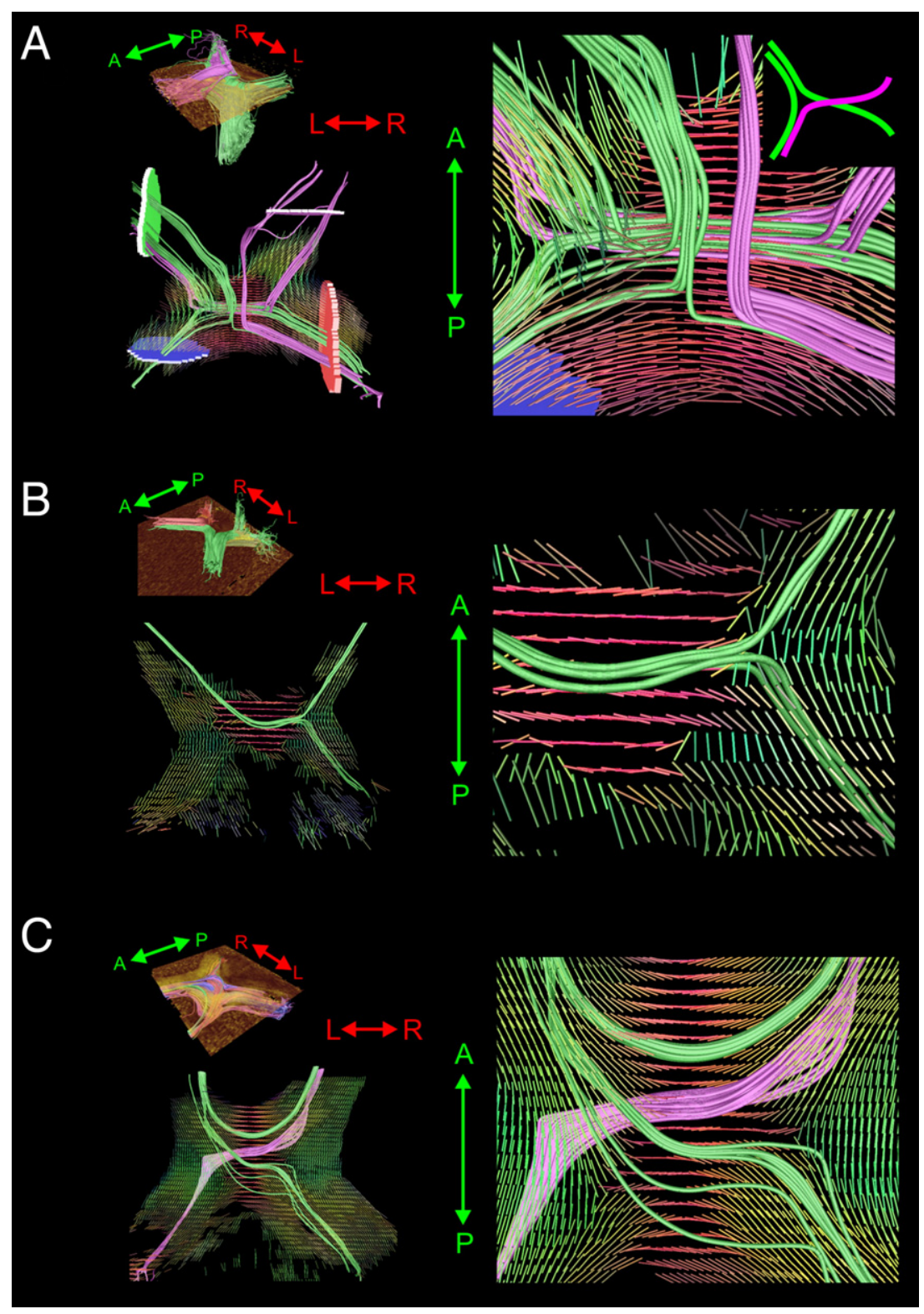

Fig. 4. A detailed rendering (from above) of selected tractography results for OC1 (A), OC2 (B) and OC3 (C). Top left inset of each shows a 3D rendering of a subset of tracked streamlines though each chiasm, along with the position of a near-axial slice. The left column shows a direction color-coded rendering of local primary diffusion directions in the selected slice along with tracked streamlines passing through critical regions in the slice. The right column shows an enlargement of the critical regions in the slice. Coloring of seed regions and tractography results as before. Inset at the top right shows a schematic of the expected true course of the local fibers. Note that only parts of the rendered tracts run through the selected slices.

the inferior-superior dimension) at a reasonably large angle and do not pass through the critical branching points.

The effects of spatial resolution on the results were investigated by performing the same analysis on down-sampled versions of OC3. OC3 was selected because it showed the most consistent percentage of tracked fibers crossing over the midline from optic nerve to optic tract. Absolute numbers and relative percentages of tracked fibers with the left or right optic nerve ROI as the seeding region for the down-sampled datasets are listed in Table 2. Observing the proportion of tracked fibers for OC3 with a given termination area with progressively coarser resolution in Tables 1 and 2, two observations can be made. First, there is a clear tendency for the underrepresentation of fibers crossing from optic nerve to contra- lateral optic tract ROI to get more severe with decreasing resolution. The percentage of optic nerve streamlines crossing to the contralateral optic tract ROI in the original data is about $5 \%$. It decreases to $4 \%, 3 \%$ and $1 \%$ in the down-sampled datasets. Second, an increase with coarser resolution in the overrepresentation of ipsilateral nerve-to-tract connections seems evident. Where the percentage of optic nerve streamlines passing to the ipsilateral optic tract ROI in the original data is about $60 \%$, it increases to $65 \%, 70 \%$ and $80 \%$ in the down-sampled datasets. This increase in streamlines that remain on one side of the midline is gained not only from decreased tracings from nerve to contralateral tract, but also from a decreasing proportion of nerve-to-nerve connections. All streamlines resulting from the fiber trackings from the optic nerve ROIs, 
Table 2

Absolute numbers and percentages (in brackets) of streamlines tracked from a given seed location (row) that passes through a given target location (column) for the three down-sampled versions of OC3

\begin{tabular}{|c|c|c|c|c|c|}
\hline & & Left nerve & Right nerve & Left tract & Right tract \\
\hline \multicolumn{6}{|c|}{$1 \times 2 \times 2(312.5 \mu \mathrm{m})$} \\
\hline Left nerve & $(4053)$ & - & $596(31 \%)$ & $1253(65 \%)$ & $75(4 \%)$ \\
\hline Right nerve & (3736) & $700(34 \%)$ & - & $85(4 \%)$ & $1299(62 \%)$ \\
\hline \multicolumn{6}{|c|}{$2 \times 4 \times 4(625 \mu \mathrm{m})$} \\
\hline Left nerve & $(2707)$ & - & $361(28 \%)$ & $918(70 \%)$ & $33(2 \%)$ \\
\hline Right nerve & $(2695)$ & $466(32 \%)$ & - & $63(4 \%)$ & $941(64 \%)$ \\
\hline \multicolumn{6}{|c|}{$4 \times 8 \times 8(1250 \mu \mathrm{m})$} \\
\hline Left nerve & $(2145)$ & - & $143(18 \%)$ & $656(82 \%)$ & $3(0.3 \%)$ \\
\hline Right nerve & $(2266)$ & $185(16 \%)$ & - & $12(1 \%)$ & $945(83 \%)$ \\
\hline
\end{tabular}

Percentages are relative to all streamlines that reached a location (i.e. relative to the sum of streamlines in a row). The absolute number of lines tracked from the seed location (longer than $2 \mathrm{~mm}$ ) is listed in brackets.

passing through the contralateral optic tract ROIs, are shown in Fig. 5. A second observation, besides the decreasing number of the crossing nerve-to-tract connections with decreasing resolution, also evident from the decreasing percentages in Table 2, is that the exact path followed by those connections also seems to alter. The crossing connections in totality seem to pass through a successively narrower portion of the central chiasm. Furthermore, the tracts that remain at the lowest resolutions predominantly have a straight left-to-right path through the central chiasm. Fibers curving back towards the contralateral optic nerve, before bending sharply posteriorly towards the contralateral optic tract, are soon smoothed to a seemingly more direct (but not more veridical, see Fig. 1) left-to-right path through the chiasm. Interpolation of the down-sampled datasets to the original resolution for easy visual comparison shows partial volume averaging in the coarser resolution datasets to be the cause of these results. The subtle anterior-posterior slope of the fibers crossing through the central chiasm is progressively averaged to an almost pure left-to-right orientation of the eigenvectors over more and more of the area surrounding the midline in the lower resolution datasets. Furthermore, partial volume averaging of the central chiasm with parts of the incoming and outgoing optic nerves and tracts causes the narrowing of the representation of the central chiasm at lower spatial resolution.

\section{Discussion}

High-resolution diffusion tensor imaging of the human optic chiasm was performed ex vivo at ultra-high field to evaluate the

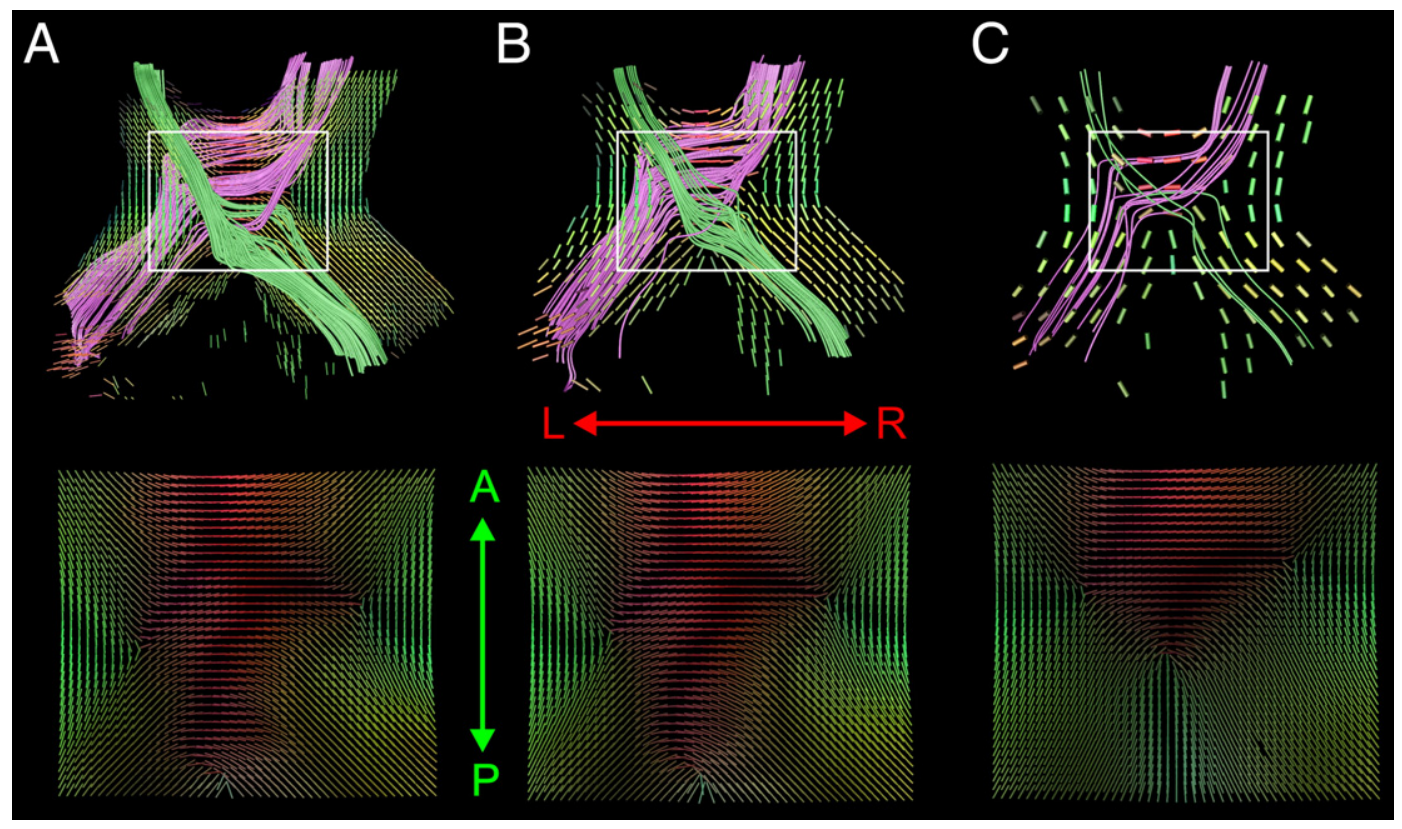

Fig. 5. Top row: results for fiber tracking from the optic nerve ROIs, passing through the contralateral optic tract ROIs for each of the down-sampled versions of the OC3 dataset. Tracked streamlines are overlaid on a cylinder rendering of primary axes of diffusion in a near-axial slice through the middle of the chiasm. Bottom row: a resampling (by interpolation) of part of the selected slice of each down-sampled dataset to the original resolution of $156 \mu \mathrm{m}$ to illustrate the loss of spatial detail at a progressively coarser resolution. 
performance of the diffusion tensor model and streamline tractography algorithms. The complex micro-structure of the optic chiasm where complex fiber configurations are present, such as kissing, crossing, convergence and sharp curves, was well characterized at the acquired resolution. Specifically, the regions within the optic chiasm where such problematic configurations occur could be localized with high spatial accuracy. Two zones within the central chiasm, in particular, contain most of the complex fiber configurations. The first is located at the lateral-most aspects of the central chiasm where it intersects with the medial aspects of the optic nerves and tracts and is highlighted by a sharp reduction in the $C_{1}$ coefficient, indicating non-prolate estimated tensor shapes there. The second region of complex fiber architecture is the expected crossing of tracts from the two optic nerves decussating to their contralateral optic tracts in the central optic chiasm. The diffusion tensor model is unable to accurately represent this crossing, resulting from its Gaussian approximation of the diffusion profile. Most likely the average direction of the two crossing fibers is what is represented at the crossing sites in the central chiasms.

Streamline tractography by a line propagation algorithm was used to investigate to which degree the known macroscopic connectivity through the optic chiasm could be reproduced. Overall, fibers crossing from optic nerve to contralateral optic tract, which should be the most numerous, are underrepresented, whereas fibers connecting ROIs on the same side of the horizontal or vertical midline are heavily overrepresented. The overrepresentation of tracts connecting optic nerve with the ipsilateral optic tracts and connections between the two nerves or between the two tracts are mainly the result of problems of the tracking algorithm at the two regions of complex fiber architecture.

Down-sampling of the original datasets to simulate lower resolution acquisitions showed importance of acquisition at a high spatial resolution. The underrepresentation of fibers crossing from optic nerve to contralateral ROI and the overrepresentation of ipsilateral nerve-to-tract connections got more severe with decreasing resolution. Moreover, the actual paths traced out in the downsampled datasets for the remaining crossing fiber were seen to be less veridical. These distorted paths seem to be caused by the distorted tensor fields resulting from partial volume averaging in the lower resolution datasets. A question that arises is how well lower resolution acquisitions are approximated by spatial averaging of a high-resolution dataset. Presumably, the partial volume averaging that occurs in larger voxels, which is of most interest in this study, is well approximated by the averaging of smaller voxels. However, an actual acquisition at a lower resolution will have more consequences. Particularly, dropouts due to field inhomogeneities and susceptibility-related artifacts will increase at lower spatial resolutions. This is not reflected in the simple averaging of voxels acquired at a high resolution. Therefore, the averaging used here is likely to give a 'best-case' simulation of a lower resolution dataset, where the quality of a real equivalent is expected to be affected by a higher degree of distortions and drop-outs. A future experiment with acquisitions at multiple resolutions would be interesting to pursue this point. Furthermore, in vivo scans at the currently attainable resolutions would be a useful complement to assess tractography in clinical conditions.

Overall, this study shows the post-mortem investigation of fixed tissue samples with DTI at high field strengths to be an attractive candidate for the evaluation of MR-based diffusion models and tractography algorithms and the effects of spatial resolution on their performance. Moreover, even without tractography, the mapping of characteristics of local diffusion in these datasets can potentially increase our knowledge of the detailed 3D anatomy of neural structures, such as the human optic chiasm. A limitation of the current study is the lack of a ground truth for the exact true paths followed by axonal fiber bundles through the chiasm. Although much of the macroscopic connection through the chiasm and even the general shapes of the paths through the chiasm is known (see Fig. 1), decisions on the correctness of discrete tracked streamlines in individual chiasms need more information. A tracked path could seem compatible with prior knowledge and the data at hand, yet be non-veridical. Combination of high-resolution imaging with more classical ways of determining anatomical connectivity, such as tracer injections, seems the most suitable way of providing a gold standard for the validation of tractography results (see e.g. Dauguet et al., 2006)

Some of the obvious errors made by the DTI-based line propagation algorithm clearly arise from the inadequacy of the diffusion tensor model to represent complex fiber architecture, such as fiber crossings, and from the constraint on the tractography algorithm to use only local information. Complex fiber configurations, such as crossings, when captured in a single voxel, will give rise to non-Gaussian diffusion profiles due to partial volume averaging (Alexander et al., 2002; Frank, 2001, 2002; Tuch et al., 2002). The diffusion tensor is unable to represent such profiles with more than a single defined maximum, as illustrated, for example, in the case of the low-angle crossing in the central optic chiasm. Recently, alternative models, capable of representing non-Gaussian diffusion profiles in voxels with complex fiber architectures, have been proposed (Assaf et al., 2004; Jansons and Alexander, 2003; Liu et al., 2004; Ozarslan and Mareci, 2003; Peled et al., 2006; Tournier et al., 2004; Tuch, 2004; Tuch et al., 2003; Wedeen et al., 2005). The estimation of these models requires high angular resolution diffusion imaging (HARDI) acquisitions, where the angular resolution of the diffusion direction sampling is greatly increased, from six directions, needed for estimation of the diffusion tensor, to tens or even hundreds of directions. It will be interesting to investigate whether these non-Gaussian diffusion models are capable of representing the diffusion profiles in regions of complex architecture in the optic chiasm. Furthermore, it will be informative to evaluate tractography results based on these models in order to verify whether a more accurate local representation of diffusion leads to more accurate tracked global connections.

The constraint of line propagation algorithms to only use local information at the current point of tracking has recently led to two further forms of developments in tractography algorithms. These developments have aimed at removing two constraints of the line propagation algorithm: its use of only local information at the current tracking point and its lack of providing any kind of reliability measure for a resulting streamline. First, some developments have taken place in tractography algorithms that minimize a more global fit of a streamline to the discrete estimated tensor field through which it passes, for instance by estimating geodesics in the tensor field considered as a Riemannian manifold (Lenglet et al., 2004) or by using the Metropolis algorithm, a simulated annealing approach that iteratively maximizes the fit of the entire tract to the data (Tuch, 2002). This could make the resulting streamlines less prone to errors at critical points such as crossings or regions of high curvature. Second, recently several groups have developed so-called probabilistic tractography algorithms that provide for a given seedpoint not a single most likely terminal point of connection along with a joining tract, but rather a 3D map of connection probability. The idea 
is to provide a probabilistic index of connectivity (PICo) that quantifies the likelihood of an anatomical connection linking two points given the estimated field of local diffusion profiles (Parker et al., 2003). Such probabilistic tractography algorithms generally involve either a Monte Carlo sampling scheme where many repetitions of a diffusing particle following a noise-perturbed path are summarized in a map of relative 'particle visits' (Behrens et al., 2003; Hagmann et al., 2003; Koch et al., 2002; Parker and Alexander, 2003; Parker et al., 2003) or some form of a front evolution algorithm that will assign arrival times to all points in the volume, interpretable as the inverse of connection probability (Campbell et al., 2005; Jackowski et al., 2005; Parker et al., 2002).

Investigation of the performance of these alternative algorithms on high-resolution datasets such as those used here will be of considerable interest. Line propagation algorithms use only local information at each step through the data, which can lead to rapid accumulation of errors, particularly when critical regions (crossings, high curvature) have to be passed. Potentially, algorithms that maximize the global fit of a trajectory are less prone to errors at critical regions when a wrong path through a critical region will decrease the fit to the surrounding data. It should be noted, however, that the constraints of surrounding data do not always resolve the correct passage through a critical region, as is the case, for instance in a kissing vs. crossing situation. The application of probabilistic algorithms to the current datasets could be of interest to investigate whether the connection likelihoods they provide would reflect the relative proportions of streamline terminations for a given seed ROI reported here. The evolution of the path of a randomly perturbed particle or a propagating front is generally constrained by the same local information at the point of propagation (shapes of estimated tensors) and the same constraints (anisotropy thresholds, angle thresholds) as the standard deterministic line propagation algorithms are. Therefore, further investigation is needed to determine whether switching from a deterministic to a stochastic tractography algorithm will provide an improvement in the accuracy of the tracking results.

This study has shown that the post-mortem investigation of fixed tissue samples with DTI at high field strengths is an attractive candidate for the evaluation of MR-based diffusion models and tractography algorithms. Furthermore, the effects of spatial resolution and the varying degrees of partial volume averaging of complex fiber architecture on the performance of these methods could be investigated. Diffusion MR-based tractography has the potential to provide quantitative measures of axonal connectivity in the living human brain. Future investigations of the performance of MR-based diffusion models and tractography algorithms on fixed tissue samples, in combination with passively diffusing tracers, is of great importance in the validation of this endeavor.

\section{Acknowledgments}

The authors would like to thank Dr. Kamil Ugurbil for enabling the measurements at the CMRR. This work was supported by NIH grants RR08079, the Human Frontiers Science Program (AR, DSK, RG) and the Keck Foundation.

\section{References}

Alexander, A.L., Hasan, K.M., Lazar, M., Tsuruda, J.S., Parker, D.L., 2001. Analysis of partial volume effects in diffusion-tensor MRI. Magn. Reson. Med. 45, 770-780.

Alexander, D.C., Barker, G.J., Arridge, S.R., 2002. Detection and modeling of non-Gaussian apparent diffusion coefficient profiles in human brain data. Magn. Reson. Med. 48, 331-340.

Assaf, Y., Freidlin, R.Z., Rohde, G.K., Basser, P.J., 2004. New modeling and experimental framework to characterize hindered and restricted water diffusion in brain white matter. Magn. Reson. Med. 52, 965-978.

Basser, P.J., Mattiello, J., LeBihan, D., 1994. Estimation of the effective selfdiffusion tensor from the NMR spin echo. J. Magn. Reson., B 103, 247-254

Basser, P.J., Pajevic, S., Pierpaoli, C., Duda, J., Aldroubi, A., 2000. In vivo fiber tractography using DT-MRI data. Magn. Reson. Med. 44, 625-632.

Beaulieu, C., 2002. The basis of anisotropic water diffusion in the nervous system - a technical review. NMR Biomed. 15, 435-455.

Behrens, T.E., Woolrich, M.W., Jenkinson, M., Johansen-Berg, H., Nunes, R.G., Clare, S., Matthews, P.M., Brady, J.M., Smith, S.M., 2003. Characterization and propagation of uncertainty in diffusion-weighted MR imaging. Magn. Reson. Med. 50, 1077-1088.

Campbell, J.S., Siddiqi, K., Rymar, V.V., Sadikot, A.F., Pike, G.B., 2005. Flow-based fiber tracking with diffusion tensor and q-ball data: validation and comparison to principal diffusion direction techniques. NeuroImage 27, 725-736.

Catani, M., Howard, R.J., Pajevic, S., Jones, D.K., 2002. Virtual in vivo interactive dissection of white matter fasciculi in the human brain. NeuroImage 17, 77-94.

Conturo, T.E., Lori, N.F., Cull, T.S., Akbudak, E., Snyder, A.Z., Shimony, J.S., McKinstry, R.C., Burton, H., Raichle, M.E., 1999. Tracking neuronal fiber pathways in the living human brain. Proc. Natl. Acad. Sci. U. S. A. 96, 10422-10427.

Dauguet, J., Peled, S., Berezovskii, V., Delzescaux, T., Warfield, S.K., Born, R., Westin, C.F., 2006. 3D histological reconstruction of fiber tracts and direct comparison with diffusion tensor MRI tractography. Med. Image Comput. Comput. Assist. Interv. Int. Conf. Med. Image Comput. Comput. Assist. Interv. 9, 109-116.

Frank, L.R., 2001. Anisotropy in high angular resolution diffusion-weighted MRI. Magn. Reson. Med. 45, 935-939.

Frank, L.R., 2002. Characterization of anisotropy in high angular resolution diffusion-weighted MRI. Magn. Reson. Med. 47, 1083-1099.

Guilfoyle, D.N., Helpern, J.A., Lim, K.O., 2003. Diffusion tensor imaging in fixed brain tissue at 7.0 T. NMR Biomed. 16, 77-81.

Hagmann, P., Thiran, J.P., Jonasson, L., Vandergheynst, P., Clarke, S., Maeder, P., Meuli, R., 2003. DTI mapping of human brain connectivity: statistical fibre tracking and virtual dissection. NeuroImage 19, 545-554.

Horton, J.C., 1997. Wilbrand's knee of the primate optic chiasm is an artefact of monocular enucleation. Trans. Am. Ophthalmol. Soc. 95, 579-609.

Jackowski, M., Kao, C.Y., Qiu, M., Constable, R.T., Staib, L.H., 2005. White matter tractography by anisotropic wavefront evolution and diffusion tensor imaging. Med. Image Anal. 9, 427-440.

Jansons, K.M., Alexander, D.C., 2003. Persistent angular structure: new insights from diffusion MRI data. Dummy version. Inf. Process. Med. Imag. 18, 672-683.

Jones, D.K., Simmons, A., Williams, S.C., Horsfield, M.A., 1999. Noninvasive assessment of axonal fiber connectivity in the human brain via diffusion tensor MRI. Magn. Reson. Med. 42, 37-41.

Koch, M.A., Norris, D.G., Hund-Georgiadis, M., 2002. An investigation of functional and anatomical connectivity using magnetic resonance imaging. NeuroImage 16, 241-250.

Lazar, M., Weinstein, D.M., Tsuruda, J.S., Hasan, K.M., Arfanakis, K., Meyerand, M.E., Badie, B., Rowley, H.A., Haughton, V., Field, A., Alexander, A.L., 2003. White matter tractography using diffusion tensor deflection. Hum. Brain Mapp. 18, 306-321.

Lee, J.H., Tobias, S., Kwon, J.T., Sade, B., Kosmorsky, G., 2006. Wilbrand's knee: does it exist? Surg. Neurol. 66, 11-17 (discussion 17).

Lenglet, C., Deriche, R., Faugeras, O., 2004. Inferring white matter geometry from diffusion tensor MRI: application to connectivity mapping. In: Pajdla, T., Matas, J. (Eds.), 8th European Conference on Computer Vision. Springer-Verlag, Prague, Czech Republic. 
Liu, C., Bammer, R., Acar, B., Moseley, M.E., 2004. Characterizing nonGaussian diffusion by using generalized diffusion tensors. Magn. Reson. Med. 51, 924-937.

Lori, N.F., Akbudak, E., Shimony, J.S., Cull, T.S., Snyder, A.Z., Guillory, R.K., Conturo, T.E., 2002. Diffusion tensor fiber tracking of human brain connectivity: acquisition methods, reliability analysis and biological results. NMR Biomed. 15, 494-515.

Mori, S., Crain, B.J., Chacko, V.P., van Zijl, P.C., 1999. Three-dimensional tracking of axonal projections in the brain by magnetic resonance imaging. Ann. Neurol. 45, 265-269.

Mori, S., Kaufmann, W.E., Davatzikos, C., Stieltjes, B., Amodei, L., Fredericksen, K., Pearlson, G.D., Melhem, E.R., Solaiyappan, M., Raymond, G.V., Moser, H.W., van Zijl, P.C., 2002. Imaging cortical association tracts in the human brain using diffusion-tensor-based axonal tracking. Magn. Reson. Med. 47, 215-223.

Ozarslan, E., Mareci, T.H., 2003. Generalized diffusion tensor imaging and analytical relationships between diffusion tensor imaging and high angular resolution diffusion imaging. Magn. Reson. Med. 50, 955-965.

Pajevic, S., Pierpaoli, C., 1999. Color schemes to represent the orientation of anisotropic tissues from diffusion tensor data: application to white matter fiber tract mapping in the human brain. Magn. Reson. Med. 42, 526-540.

Parker, G.J., Alexander, D.C., 2003. Probabilistic Monte Carlo based mapping of cerebral connections utilising whole-brain crossing fibre information. Inf. Process. Med. Imag. 18, 684-695.

Parker, G.J., Stephan, K.E., Barker, G.J., Rowe, J.B., MacManus, D.G., Wheeler-Kingshott, C.A., Ciccarelli, O., Passingham, R.E., Spinks, R.L., Lemon, R.N., Turner, R., 2002. Initial demonstration of in vivo tracing of axonal projections in the macaque brain and comparison with the human brain using diffusion tensor imaging and fast marching tractography. NeuroImage 15, 797-809.

Parker, G.J., Haroon, H.A., Wheeler-Kingshott, C.A., 2003. A framework for a streamline-based probabilistic index of connectivity (PICo) using a structural interpretation of MRI diffusion measurements. J. Magn. Reson. Imaging 18, 242-254.

Peled, S., Friman, O., Jolesz, F., Westin, C.F., 2006. Geometrically constrained two-tensor model for crossing tracts in DWI. Magn. Reson. Imaging 24, 1263-1270.

Pierpaoli, C., Basser, P.J., 1996. Toward a quantitative assessment of diffusion anisotropy. Magn. Reson. Med. 36, 893-906.

Poupon, C., Clark, C.A., Frouin, V., Regis, J., Bloch, I., Le Bihan, D., Mangin, J., 2000. Regularization of diffusion-based direction maps for the tracking of brain white matter fascicles. NeuroImage 12, 184-195.

Press, W., Teukosky, S., Vetterling, W., Flannery, B., 2002. Numerical Recipes in $\mathrm{C}++$ : The Art of Scientific Computing, 2nd ed. Cambridge Univ. Press, Cambridge.

Standring, S. (Ed.), 2005. Gray's Anatomy: The Anatomical Basis of Clinical Practise, 39 ed. Elsevier Churchill Livingstone, Edinburgh, London.

Sun, S.W., Neil, J.J., Song, S.K., 2003. Relative indices of water diffusion anisotropy are equivalent in live and formalin-fixed mouse brains. Magn. Reson. Med. 50, 743-748.

Sun, S.W., Neil, J.J., Liang, H.F., He, Y.Y., Schmidt, R.E., Hsu, C.Y., Song, S.K., 2005. Formalin fixation alters water diffusion coefficient magnitude but not anisotropy in infarcted brain. Magn. Reson. Med. 53, 1447-1451.

Tournier, J.D., Calamante, F., Gadian, D.G., Connelly, A., 2004. Direct estimation of the fiber orientation density function from diffusionweighted MRI data using spherical deconvolution. NeuroImage 23, 1176-1185.

Tuch, D.S., 2002. Diffusion MRI of Complex Tissue Structure. Division of Health Sciences and Technology. Massachusetts Institute of Technology, Boston.

Tuch, D.S., 2004. Q-ball imaging. Magn. Reson. Med. 52, 1358-1372.

Tuch, D.S., Reese, T.G., Wiegell, M.R., Makris, N., Belliveau, J.W., Wedeen, V.J., 2002. High angular resolution diffusion imaging reveals intravoxel white matter fiber heterogeneity. Magn. Reson. Med. 48, 577-582.

Tuch, D.S., Reese, T.G., Wiegell, M.R., Wedeen, V.J., 2003. Diffusion MRI of complex neural architecture. Neuron 40, 885-895.

Wakana, S., Jiang, H., Nagae-Poetscher, L.M., van Zijl, P.C., Mori, S., 2004. Fiber tract-based atlas of human white matter anatomy. Radiology 230, $77-87$.

Wedeen, V.J., Hagmann, P., Tseng, W.Y., Reese, T.G., Weisskoff, R.M., 2005. Mapping complex tissue architecture with diffusion spectrum magnetic resonance imaging. Magn. Reson. Med. 54, 1377-1386.

Westin, C.F., Maier, S.E., Mamata, H., Nabavi, A., Jolesz, F.A., Kikinis, R., 2002. Processing and visualization for diffusion tensor MRI. Med. Image Anal. 6, 93-108.

Wilbrand, H., 1926. Schema des verlaufs der sehnervenfaserrn durch das chiasma. Z. Augenheilkd. 59, 135-144.

Xue, R., van Zijl, P.C., Crain, B.J., Solaiyappan, M., Mori, S., 1999. In vivo three-dimensional reconstruction of rat brain axonal projections by diffusion tensor imaging. Magn. Reson. Med. 42, 1123-1127. 